\title{
Advanced Intracardial Biosignal Processing
}

\author{
Marek Penhaker ${ }^{1}$, Petr Klimes ${ }^{1}$, Jakub Pindor ${ }^{1}$, and David Korpas ${ }^{2}$ \\ ${ }^{1}$ VSB - Technical University of Ostrava, Faculty of Electrical Engineering and Computer \\ Science, Ostrava, Czech Republic \\ ${ }^{2}$ Silesian University, Faculty of Public, Policies Institute of Nursing, Opava, \\ Czech Republic \\ \{marek.penhaker, petr.klimes, jakub.pindor\} @vsb.cz, \\ david.korpas@seznam.cz
}

\begin{abstract}
In this work deals about the efficient intracardial ECG analysis algorithm. The main focus was design an optimal detection method for intracardial signals based on essential intracardial measuring methods and basic principles of gathering and processing data from invasive catheters. Intracardial ECG analysis is further important step in heart behavior understanding, especially it's electric manners. Detailed signal description generated in heart,together with heart function knowledge can provide us with an useful information about heart's condition or it's diseases. Designed detection methodis able to mark significant points in intracardial records, compute it's elemental parameters and important time intervals. Incurred algorithm was designed and tested on intracardial records provided by Cardiology Clinic of Hospital IKEM - Praha, and Electrophysiology Laboratory of Hospital Podlesi - Trinec.
\end{abstract}

Keywords: iECG, Detection, Filtering, Processing.

\section{Introduction}

By entering this work was, among other things, analysis and detection of significant characters in the intracardiac ECG recordings with subsequent processing of such information. Data from measurements of intracardiac ECG was provided in a hospital Podlesi Třinec. These are the signals measured at different types of system operations and subsequently Exported Cardiolab in *. txt. Each data set is also associated *. inf file that contains additional information about the record length, sampling frequency, number of leads, etc. The development application was trying to get signals of various parameters from different places with varying degrees of heart hampering to determine a success or approximate boundaries of functionality algorithm.

\subsection{Source Intracardial Data}

Data is stored in a file with the extension *. txt, and can therefore be viewed as a classic notepad in Windows. After the opening we see that this is a table of numbers, where each column represents a seduction. The measured signals were recorded with 
a sampling frequency of $977 \mathrm{~Hz}$. It follows that for such a long signal for 30 seconds for each lead-recorded 29310 samples. If recorded three intracardiac leads, for example, the first of the CS catheter ablation, and two, that the total surface leads recorded with 15 leads, it means that the file of the thirty-second measurement has 439,650 samples, or figures in the table. This comprehensive text file on your computer takes up about $5 \mathrm{MB}$ of memory.

The benefits file with the *. txt is the fact that it is very easy to load into MATLAB as a multidimensional variable. Indexing, we were able to separate each lead, respectively. vector and store it as a separate variable. Thus it can be done with all leads and each store separately under its own pre-chosen name. This prepared data from individual leads are ready for further processing.

\section{$2 \quad$ Signal Preprocessing}

In this work, the data are processed intracardiac leads see. Figure 1 shows the loaded signal from the ablation catheter accurately record the first 2.5 seconds. Signals from the chambers, which are clearly visible sign precedes the $\mathrm{P}$ wave, a signal from the hall. This wave, along with other manifestations of tissue, however, at this point redundant information. Our goal is to become independent and to detect ventricular signal.

For recognition of deformed QRS complex need to adjust the signal first. In the first phase of minor cropping potential and then using the wavelet transform.

Setting the threshold at the loaded signal seen in Figure 2 Red lines show the threshold values that are set both in positive terms and in the negative. Everything in this limited area is then ignored and reset the chart. However, it is necessary for this operation to maintain an identical length of the signal. It is therefore a direct replacement of values below the threshold at zero.

In the algorithm shown below is copied into a variable with a length of the original signal is modified in the wake.

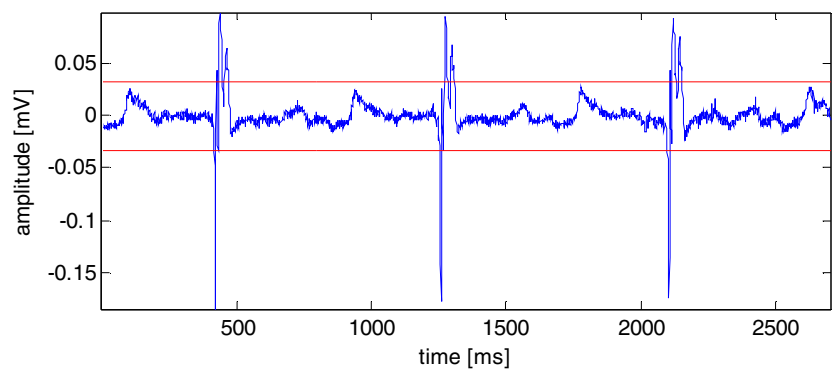

Fig. 1. From the loaded signal is displayed intracardiac catheter ablation of seduction

The next step is transformation modified signal using wavelets. Specifically, the selected wavelet bior1.5. As explained in section 4.3, determine the type of waves 
preceded the experimental procedure is based on knowledge parameters of wavelets and its use in other applications. Subsequent levels of decomposition setting, or scale, using this formula was based on wavelets for detecting QRS complexes in the surface ECG 02.05.

Finishing the signal is conducted continuous wavelet transform (CWT), wavelets bior1.5, with the fourth level of decomposition. The result of this transform shown in Figure 2.

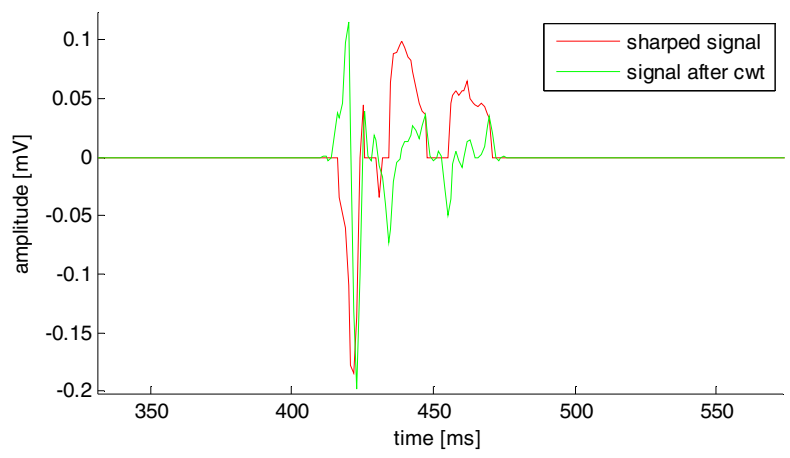

Fig. 2. Adjustment signal continuous wavelet transform - CWT, bior1.5. For clarity, only the first pick was detected excitation signal. It is clearer and shows how the signal continuous wavelet transform based on original changed shape.

\subsection{Finding Three Significant Characters in the Intracardiac Recordings}

Trimming curve prepared in advance and continuous wavelet transform is now available for the final stage of detection. This is a clear target indication of significant peaks that are detected primarily ventricular cycles and finding the beginnings and ends of these impulses. As already mentioned in Chapters 3 and 4, our task is not interested to follow more closely the morphology of the curve. Valuable data are kept in the information exactly where on the timeline is detected peak of excitement, when the excitement begins and ends, respectively. length and its relationship to neighboring vzruchům detected. First of all, we must clearly identify the detected peak of excitement.

\subsection{Finding the Detected Peak Impulse}

After adjusting signal quality before building a relatively simple task. The signal is free from all the surrounding potentials which are not interesting for us as well is wavelet transform rid of sharp transitions and changed into a form that presents Figure 3. So the only thing left, and find places where the transformed curve intersects the zero. We know that at the same time when the original curve reaches its local maximum, then the detected peak of excitement. 
The problem with this procedure is the possibility of intersection of two lines of zeros. The signal zero crossing can be upward or downward, or may go from negative to positive and vice versa. Is needed in this algorithm into account, otherwise it will only detect one case of two and a high probability of excitation peak detect.

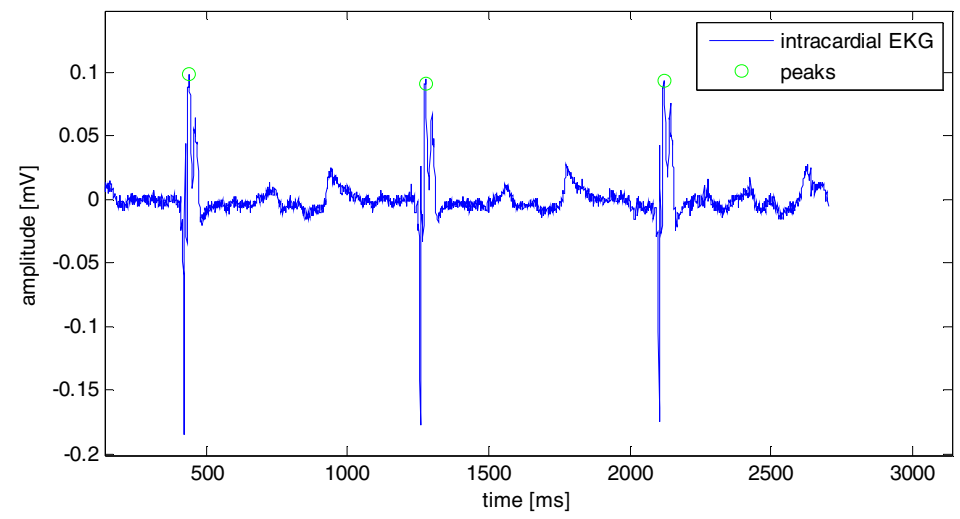

Fig. 3. Detected peaks intracardiac ECG electrical activity generated by ventricular

Another complication lies in the multiple detection. Figure 28 shows that the zero passage of the transformed signal below the top of the original passage is not only zero in the area. Algorithm to actually detect several peaks and is not clearly determined which of them is described as the pinnacle of the impulse, ie as a global summit. Assuming that it would be a simple curve with really only one local maximum to avoid the multiple detection. With this, however, can not count, especially with intracardiac leads that may have a completely unexpected

The solution to this problem is the second part of the displayed code, which provides only the label is always the highest, that is the most prominent peak, which will ensure compliance with the requirement to detect a global peak of excitement. S_cwt variable represents the signal after processing the first part of the code and wavelet transforms. In the first phase will split the signal into sections that define the detected impulses. With a condition that can be detected only one peak in each such segment, the algorithm looks for the one with the highest amplitude relative to other peaks found. The definition of this section is based on sampling frequency, while the assumption of a distribution of excitation. As a limit value for setting limits for cropping signal, this value may be modified according to user needs and current signal parameters.

In this way, peaks are detected, or excitation peaks, which are used in the subsequent detection of the beginning and end of the impulse as a starting point, clearly defining where the peak is located. 


\subsection{Finding the Beginning and End of the Detected Impulse}

The next phase of the effort comes reliable indication of the place where the detected excitation begins and ends. Early attempts to design this part of the algorithm contributed to the final solution with satisfactory results. To better understand the description and the impasse of development.

The original idea comes from practice. The doctor looks at the curve and just see the place where the excitement begins. Identifies it as that of the relatively flat line curve begins to rise suddenly to higher values. Likewise, it is also able to detect an algorithm to detect the beginning of excitement, but it has to be defined in accordance with these requirements. It is not possible based on the projected shape of the curve, as is the case with the surface ECG. The shape can take various forms, it is necessary to build on this basic premise: excitement begins where the curve starts from a relatively flat line of steep climb to higher values.

To find this place is first necessary to map the section signal. Rather it is a section from the detected peak before the beginning of oscillation. The signals are then backward from this point marked points that are stored in a matrix for subsequent calculations. Not only do we know their coordinates, or location on the timeline, we also represent the value of the signal amplitude at a given point.

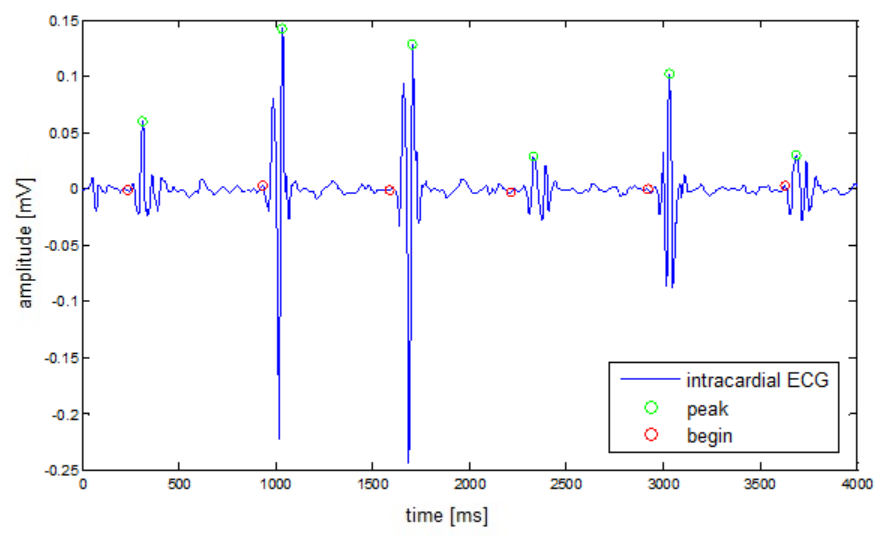

Fig. 4. Marking the beginnings of impulses detected by intracardiac ECG

This section mapped signal was then subjected to search for the smallest difference in amplitude between two neighboring points. The result of this comparison should be to find a flat end and the beginning of the steep climb. However, faced several problems. First and foremost was the smallest difference between points are often found at large distances from the actual beginning of the impulse, ie before it even began to stir. This was mainly due to the fact that the smallest difference amplitudes are often located away from the beginning of the excitement and not directly at him. The second complication occurred when the two adjacent points lie in the steep part, but one on the ascending and descending on the second, at about the same level. The algorithm then evaluate this case as a flat surface and named it as the beginning of excitement, but he saw a local maximum or minimum between these points and was 
unable to recognize that the points lie on a steep curve. Including more surrounding pixels into these calculations, the problem is solved.

To ensure a really steep marking the beginning of excitation, respectively. place before the start of the steep change was designed additional code, to ensure early recognition steep based on a predetermined level, the signal must exceed before the impulse. Points are mapped in the first phase of this calculation converted to their absolute values. It is not conclusive, if the beginning is in positive or negative values. Elements are among the neighboring points. Subsequently, the detected maximum value of the matrix marked points. This value is then determined the level of $0.1 \%$.

In the detection of all impulses to proceed similarly, but on the opposite side of the detected peak. At the same time there is an effort to find the sharp end, because the impulses are usually sharply terminated. In most cases disappear more slowly than the rise. The algorithm was therefore counted the detected displacement of the point which was found in the same manner as at the beginning of excitation.

In this way the algorithm was developed to detect peaks, start and end of detected impulses. Beginnings and endings are detected based on detecting peaks. Without a mark on top of the algorithm is able to delimit excitement. The experimental part of the algorithm was able to recognize signs and $\mathrm{Q}$ and $\mathrm{S}$ waves detected in the chamber complex. Due to the nature of the intracardiac signals, however, from this expansion was abandoned and attention was paid to quality notably through the detection of peaks of individual impulses.

\section{Analysis Result}

The algorithm was tested on approximately 75 different intracardiac signals from 15 different operations. This was how the signals from the diagnostic catheters, which provide about the best possible signal, measured directly in the heart, as well as therapeutic catheters, especially ablation, which is more therapeutic than hampering because of almost constant motion after cardiac walls.

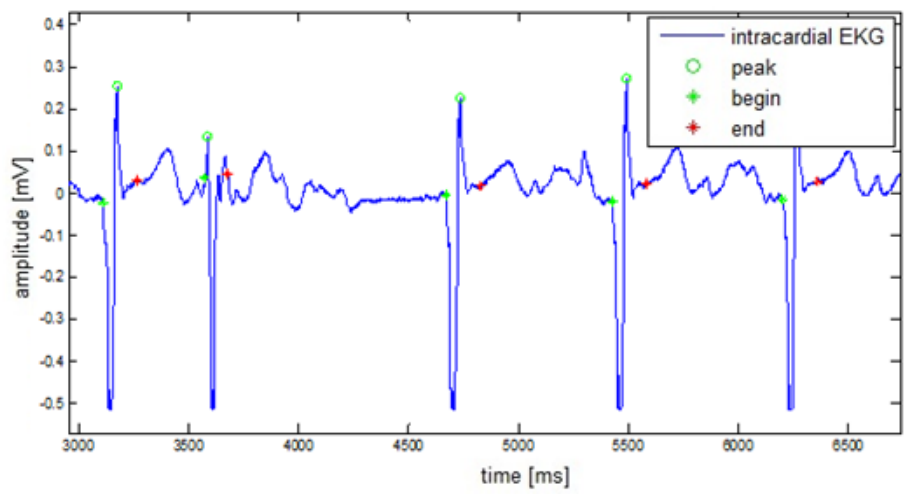

Fig. 5. Slightly hampering signal ablation catheter. Detection of peaks was flawlessly, beginnings and ends are detected for the second excitation reliably identified. 
Detection of peaks achieved when the correct setting of limits almost total success even in noisy signals. In cases where the algorithm does not detect any peaks or, conversely, create false points, the curve was so hampering that there were doubts about the actual value of the information curve.

Identifying the beginnings and ends of the excitation peaks detected in the header pipes from the CS catheter almost flawless. For noisy signals success begins to deteriorate, and to mark the beginning is always the case. Only the position of determining the beginning point is not exactly at the point of beginning of excitation.

By classifying peaks, start and end of each detected impulse was obtained by specific dates, which carry information about the amplitude of excitation, the frequency, duration, etc. The processing of this information is not targeted to any particular application. This is a demonstration of versatility of use of the algorithm. Analyzed intracardiac ECG curve has been described and this allows the subsequent use of these data for experimental and other purposes.

\section{Test Results and Evaluation by Analysis}

Of all ten measured parameters were selected to assess three basic parameters of the signal and then three time intervals for cross checking the accuracy of detection.

Table 1 are measured values from the analysis of the fifteen intracardiac signals. The upper part is shown on the measurement of diagnostic catheters, the lower part presents the signals of therapeutic catheters. HR heart rate indicates the heart rate of total recording time. It is therefore an average value in units of beats / min. The amplitude of the detected impulse Amp informs about the average height of the signal amplitude in $\mathrm{mV}$ units.

Table 1. The measured values

\begin{tabular}{|c|c|c|c|c|c|c|}
\hline \multicolumn{7}{|c|}{ Signals from a diagnostic catheter EN } \\
\hline & HR [bpm] & Amp [mV] & $P-P[\mathrm{~ms}]$ & $B-B[\mathrm{~ms}]$ & $E-E[\mathrm{~ms}]$ & Hit Rate [\%] \\
\hline 1 & 72 & 1,024 & 838 & 838 & 838 & 100 \\
\hline 2 & 72 & 0,5917 & 838 & 838 & 838 & 100 \\
\hline 3 & 90 & 0,4053 & 667 & 667 & 667 & 97,93 \\
\hline 4 & 87 & 0,4996 & 686 & 686 & 686 & 100 \\
\hline 5 & 75 & 0,4566 & 798 & 798 & 798 & 98,2 \\
\hline \multicolumn{7}{|c|}{ Signals from therapeutic catheter ABL } \\
\hline & HR [bpm] & Amp [mV] & $P-P[\mathrm{~ms}]$ & $B-$ B [ms] & $E-E[\mathrm{~ms}]$ & Hit Rate [\%] \\
\hline 1 & 74 & 0,2676 & 806 & 804 & 806 & 100 \\
\hline 2 & 106 & 0,512 & 567 & 565 & 567 & 75 \\
\hline 3 & 133 & 0,3798 & 452 & 453 & 452 & 95 \\
\hline 4 & 133 & 0,3798 & 452 & 453 & 452 & 94,04 \\
\hline 5 & 86 & 0,469 & 698 & 698 & 698 & 100 \\
\hline 6 & 86 & 0,4296 & 698 & 699 & 698 & 91,3 \\
\hline 7 & 86 & 0,6738 & 796 & 796 & 796 & 100 \\
\hline 8 & 66 & 0,4559 & 910 & 910 & 910 & 86,36 \\
\hline 9 & 85 & 1,7146 & 706 & 706 & 706 & 98,46 \\
\hline 10 & 71 & 1,2949 & 846 & 846 & 846 & 95,38 \\
\hline
\end{tabular}


The following three values of $\mathrm{PP}, \mathrm{BB}, \mathrm{EE}$ is dedicated to time intervals to assess the mutual time between detected points. This is the time interval between two adjacent peaks, the time interval between the starts of two adjacent impulses, and the interval between the end in units of ms.

The last column lists the values of hit rate achieved success at the individual signals. Word of success in this case think successfully found and marked points of the beginning, peak and end of detected impulses. This value was calculated based on detection by visual inspection for each of the records. The number of unsuccessfully marked or completely unmarked points and the total number of impulses detected catheter has been derived detection rate as a percentage.

\section{$5 \quad$ Signal Processing Ranking}

This value has a major impact on all previous calculations. If success is very small, we mean below $50 \%$, does not have a previous column, too deal with. Measured and calculated data in such a case would probably not representative. Values above $90 \%$ success rate on the contrary we affirm the accuracy of the calculated parameters.

Percentage success was calculated based on visual inspection of each detected impulse. In the event that the detector has not identified any excitement, the points were its peak, the beginning and end labeled as defective. Likewise, if the detector is evaluated as a potential suitable to describe excitement, while the excitement was not in effect, create a false detection, and also this point is marked as defective. Subsequently, the bad points of detection compared with the total number of impulses, which had a detector to recognize and label.

In the event that marked all the impulses in the signal, its peaks, starts and ends at the same time did not create any false detection, the program has reached the maximum, a $100 \%$ success rate. In case of omission of some important points, or marking the wrong places, the success rate has declined in the worst case up to $75 \%$.

Overall, the table shows that the measured signal quality diagnostic catheters is generally achieved a higher success rate, while the ablation catheter, which is often hard to read the signal, detection rate was worse.

Simultaneously with this measurement was performed peak detection statistics of success and detection of beginnings and endings, which is not included in the table. In this measurement is not take into account the type of signal. It did not matter then whether the signal from the therapeutic or diagnostic catheter is taken into account only well or poorly marked points under visual control again seen as successful or faulty. The resulting, average values of success are as follows:

The success of the algorithm for detection of significant characters in the intracardiac signals. Detection rate peaks: $98.85 \%$, The success of detection of beginnings and endings: $95.05 \%$, The success rate in detecting signals from diagnostic catheters: $99.22 \%$, The success rate in detecting signals of therapeutic catheters: $93.55 \%$. 


\section{Conclusion}

The benefit of this work is a new approach to detect points in the ECG signals nonstandard shapes. It introduced an efficient method to recognize, label and border impulses, which are directly from the heart wall observed invasive catheters. This project was created to continue work to further improve the algorithm to detect and use this system to find deposits of arrhythmia in the heart, using calculations of intervals between impulses of two different signals in real time. For processing and design of detection algorithms were used MATLAB, in which they were implemented as mathematical signal processing operations, so statistical analysis of test results.

Acknowledgment. The work and the contribution were supported by the project: Ministry of Education of the Czech Republic under Project 1M0567 "Centre of Applied Cybernetics", Student grant agency SV 4501141 "Biomedical engineering systems VII" and TACR TA01010632 "SCADA system for control and measurement of process in real time". Also supported by project MSM6198910027 Consuming Computer Simulation and Optimization. This paper has been elaborated in the framework of the IT4Innovations Centre of Excellence project, reg. no. CZ.1.05/1.1.00/02.0070 supported by Operational Programme 'Research and Development for Innovations' funded by Structural Funds of the European Union and state budget of the Czech Republic.

\section{References}

[1] Alfaouri, M., Daqrouq, K.: ECG signal denoising by wavelet transform thresholding. American Journal of Applied Sciences 5(3), 276-281 (2008)

[2] Laguna, P., Jané, R., Caminal, P.: Automatic detection of wave boundaries in multilead ECG signal: Validation with the CSE Database. Computers and Biomedical Research 27, 45-60 (2004)

[3] Vašíčková, Z., Penhaker, M., Augustynek, M.: Using Frequency Analysis of Vibration for Detection of Epileptic Seizure. In: Dössel, O., Schlegel, W.C. (eds.) WC 2009. IFMBE Proceedings, vol. 25/IV, pp. 2155-2157. Springer, Heidelberg (2009)

[4] Krejcar, O., Janckulik, D., Motalova, L.: Complex Biomedical System with Mobile Clients. In: Dössel, O., Schlegel, W.C. (eds.) WC 2009. IFMBE Proceedings, vol. 25/V, pp. 141-144. Springer, Heidelberg (2009)

[5] Prauzek, M., Penhaker, M.: Methods of comparing ECG reconstruction. In: 2nd Internacional Conference on Biomedical Engineering and Informatics, Stránky, pp. 675-678. Tianjin University of Technology, Tianjin (2009) ISBN: 978-1-4244-4133-4, IEEE Catalog number: CFP0993D-PRT 